\title{
Extensive Heterotopic Ossification Following Anterior Cervical Fusion in a Patient with Diffuse Idiopathic Skeletal Hyperostosis
}

\begin{abstract}
Dong-uhn Lee ${ }^{1}$, Hak-cheol Ko ${ }^{1}$, Byung-chul Son ${ }^{1,2}$
${ }^{1}$ Department of Neurosurgery, Seoul St. Mary's Hospital, College of Medicine, The Catholic University of Korea, Seoul, ${ }^{2}$ Catholic Neuroscience Institute, College of Medicine, The Catholic University of Korea, Seoul, Republic of Korea

Corresponding author: Byung-chul Son Department of Neurosurgery, Seoul St. Mary's Hospital, Catholic Neuroscience Institute, College of Medicine, The Catholic University of Korea, 222 Banpo-daero, Seocho-gu, Seoul 06591, Republic of Korea

Tel: $+82-2-2258-6122$

Fax: +82-2-594-4248

E-mail: sbc@catholic.ac.kr

Occurrence of heterotopic ossification $(\mathrm{HO})$ following cervical total disc replacement (TDR) is well-known phenomenon. However, its occurrence after anterior cervical fusion (ACF) is very rare. We report a 59-year-old male patient with diffuse idiopathic skeletal hyperostosis (DISH) presenting with dysphagia due to unusually rapid regrowth of cervical syndesmophytes 64 months following ACF (C3-C6) for cervical spine fracture. Indeed, development of recurrent cervical osteophytes at C4 was evident radiologically at postoperative one year and continued regrowth occurred over years. Surgeons should continue to follow patients with cervical osteophytes associated with DISH to assess the regrowth of osteophytes.
\end{abstract}

Received: February 27, 2018

Revised: March 24, 2018

Accepted: March 29, 2018
Key Words: Deglutition disorders; Hyperostosis, diffuse idiopathic skeletal; Ossification, heterotopic; Spinal fusion

\section{INTRODUCTION}

Diffuse idiopathic skeletal hyperostosis (DISH) is a common skeletal disorder in the elderly population and is characterized by flowing anterolateral ossifications alongside the right thoracic spine, frequently accompanied by similar ossifications of the remaining spinal segments and peripheral tendinous insertion ${ }^{4,8,10,12,13)}$. Although generally asymptomatic, large cervical anterior osteophytes have been reported to cause swallowing disorders ${ }^{11,14)}$. Surgical resection of the osteophytes was reported to be an effective treatment. However, postsurgical recurrence of cervical osteophytes causing dysphagia with longterm follow-up has also been reported ${ }^{11)}$. A regrowth rate of $1 \mathrm{~mm} /$ year after surgical resection of osteophytes in 20 segments of 7 patients with DISH was reported.

Heterotopic ossification (HO) refers to bone formation in tissues that normally do not exhibit osteogenesis ${ }^{15)}$. HO is a well-known complication of total hip arthroplasty (THA) and its incidence varies greatly from $2 \%$ to $53 \%$ following $\mathrm{THA}^{5,15)}$. It has also attracted clinical attention since the introduction of total disc replacement (TDR), which was designed to preserve segmental motion and theoretically prevent adjacent segment degeneration of the cervical spine following surgery for cervical spondylosis and disc disease ${ }^{5,15)}$. However, the incidence and clinical implications of $\mathrm{HO}$ following anterior cervical fusion (ACF) in patients with DISH has not been investigated. We report a case of extensive $\mathrm{HO}$ following $\mathrm{ACF}$ in a patient with DISH

\section{CASE REPORT}

A 59-year-old male patient presented with chronic refractory pain associated with bilateral arm and leg following cervical spinal cord injury. He had sustained a motor vehicle accident five years ago and became quadriplegic due to cervical cord contusion at C4 level (upper extremities, NMT grade II/lower extremities, grade 1). He underwent decompression and ACF from C3 to C6 five days after the accident. His neurologic condition did not improve postoperatively. He was rehabilitated in several hospitals, subsequently. Persistent and chronic neuropathic pain triggered by spinal cord injury developed within a month after cervical spinal trauma. The pain was described as mainly stabbing and cold with a severity of 8 to 9 out of 10 on a numerical rating scale ranging from 0 to 10 . It was not aggravated with neck motion. Spontaneous cold pain was detected in his bilateral hand and fingers, upper thoracic area, and bilateral legs. Mechanical and cold allodynia was present in his bilateral palms and fingers, consistent with at-level neuropathic pain. Chronic at-level and below-level neuropathic pain was not relieved with maximal medical treatment including 
gabapentin (1,800 mg/day), pregabalin (450 mg/day), tramadol (300 mg/day), amitriptyline (20 mg/day), and OxyContin (40 mg/ day) and transdermal fentanyl $(50 \mathrm{mcg} /$ day $)$. He was referred to our department for pain control.

He showed typical features of complete spinal cord injury at $\mathrm{C} 4 / 5$ at the time of presentation (64 months postinjury). He could not manage his routine daily activities, including eating, washing, and moving. Caregiver assistance was needed in most of his activities of daily living. His medical history was unremarkable, including diabetes, gout, and so on. During examination, he complained of chronic swallowing difficulty since the accident and the operation. Indeed, despite of several years of rehabilitation treatment, his swallowing difficulty was not improved. He has had difficulty in swallowing solid food after the accident and ACF. No evidence of airway obstruction was evident. His cervical x-ray examination at the time of presentation showed a prominent anterior cervical osteophyte at the level of $\mathrm{C} 4 / 5$ (Fig. 1). Computed tomographic (CT) scan of the neck showed a large anterior cervical osteophyte growing from the C4 corpectomy site to the right hyoid bone. Due to large osteophyte, the wall of posterior pharyngeal space was displaced medially. Pseudoarthrosis was found between the osteophyte and hyoid bone. Retrospective evaluation of DISH and $\mathrm{HO}$ was performed. The CT scan of the thoracic spine showed a longitudinal osteophyte along the right anterolateral vertebral column, consistent with DISH (Fig. 1)

Preoperative cervical spine X-ray and CT scan showed the presence of large osteophyte from $\mathrm{C} 4$ to $\mathrm{C} 7$, which was consistent with DISH, and ossified posterior longitudinal ligament (OPLL) along C4 to C6 with resultant cervical spinal stenosis (Fig. 2). Resection of large anterior cervical osteophyte from DISH and decompression of cervical spinal cord with removal of OPLL was performed by C4 corpectomy with C4 to C6 interbody fusion with mesh cage and an additional anterior cervical discectomy and fusion at $\mathrm{C} 6 / 7$. The large anterior osteophyte was successfully via cervical anterior decompression and fusion (Fig. 2). However, in the cervical x-ray obtained one year after the ACF, regrowth of the anterior cervical osteophyte was evident at the $\mathrm{C} 4$ level (Fig. 2). A follow-up lateral $\mathrm{x}$-ray of the cervical spine 32 months after the ACF revealed more extensive anterior growth of osteophyte abutting the hyoid bone, revealing a pseudoarthrosis (Fig. 2). Regrowth of the anterior osteophyte from DISH appears to have started after the ACF and with progressive enlargement.

Due to progressive growth of the osteophyte and presence of continued swallowing difficulty over 5 years after ACF, surgical resection was considered. However, the patient refused to undergo surgery of the cervical spine and regular follow-up was planned. The chronic neuropathic pain responded positively ( $>50 \%$ pain relief) with intrathecal infusion of morphine sulfate. After repeated trial of intrathecal infusion of morphine and confirmation of treatment efficacy, permanent implantation of intrathecal drug delivery system was performed. The patient
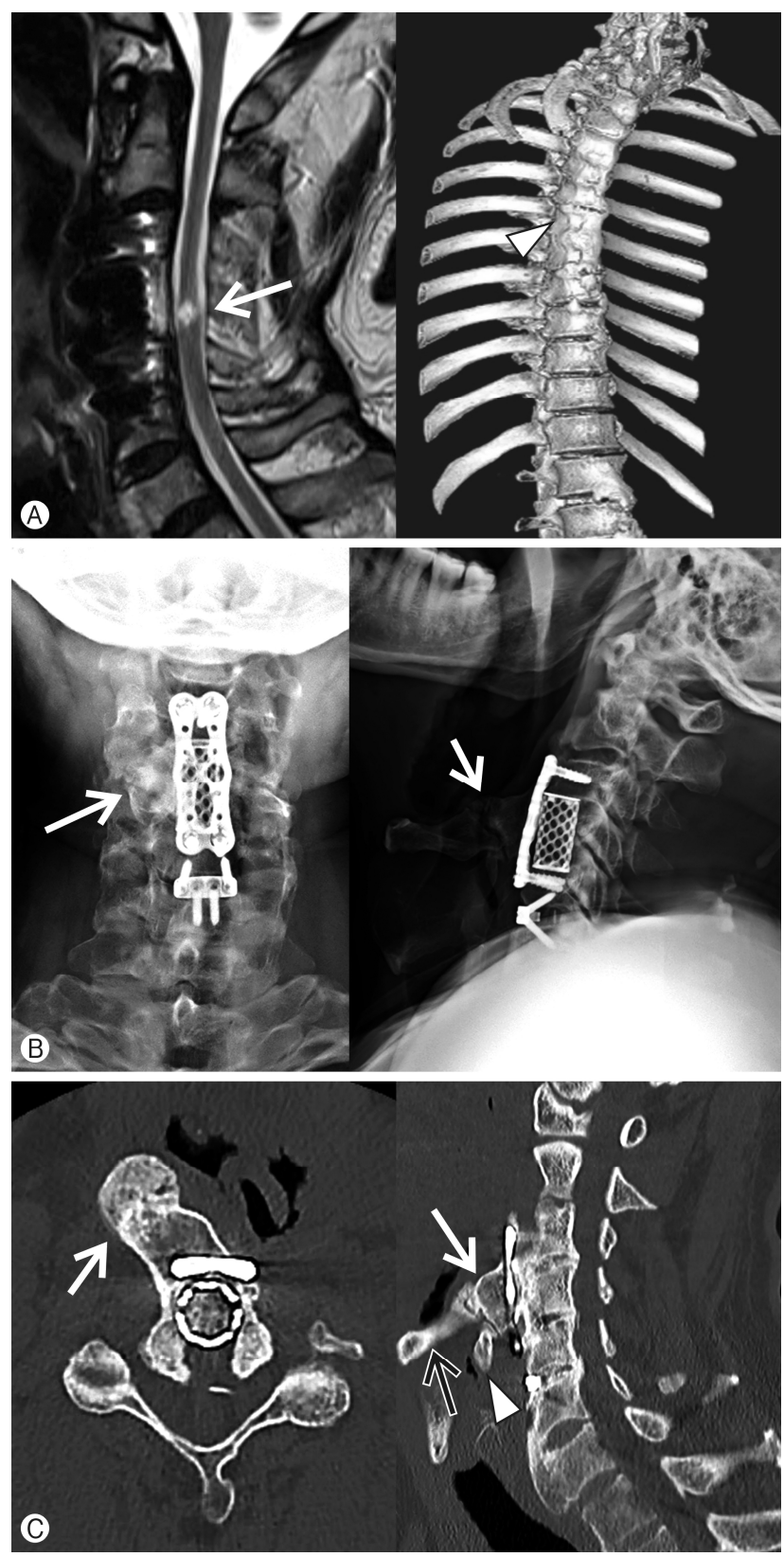

Fig. 1. Radiological findings of C4/5 spinal cord injury and heterotopic ossification (HO) following anterior cervical fusion (ACF). (A) A sagittal T2-weighted magnetic resonance imaging image (left) showing a high signal intensity lesion (arrow) within C4/5 spinal cord and a reconstructed 3-dimensional computed tomography (CT) image showing evidence (arrowhead) of diffuse idiopathic skeletal hyperostosis in the anterolateral thoracic spine. (B) Anterior-posterior (left) and lateral (right) $\mathrm{x}$-ray images showing a prominent $\mathrm{HO}$ in the right side of cervical spine following ACF. Evidence of C4 corpectomy with anterior instrumented fusion from C3 to C6 is observed. (C) Axial (left) and sagittal (right) CT images of the cervical spine. The large osteophyte of $\mathrm{HO}$ (white arrows) extends from C4/5 fusion level to the hyoid bone (black arrow) and the thyroid lamina (white arrowhead). The posterior wall of the hypopharynx is deviated to the left side. 


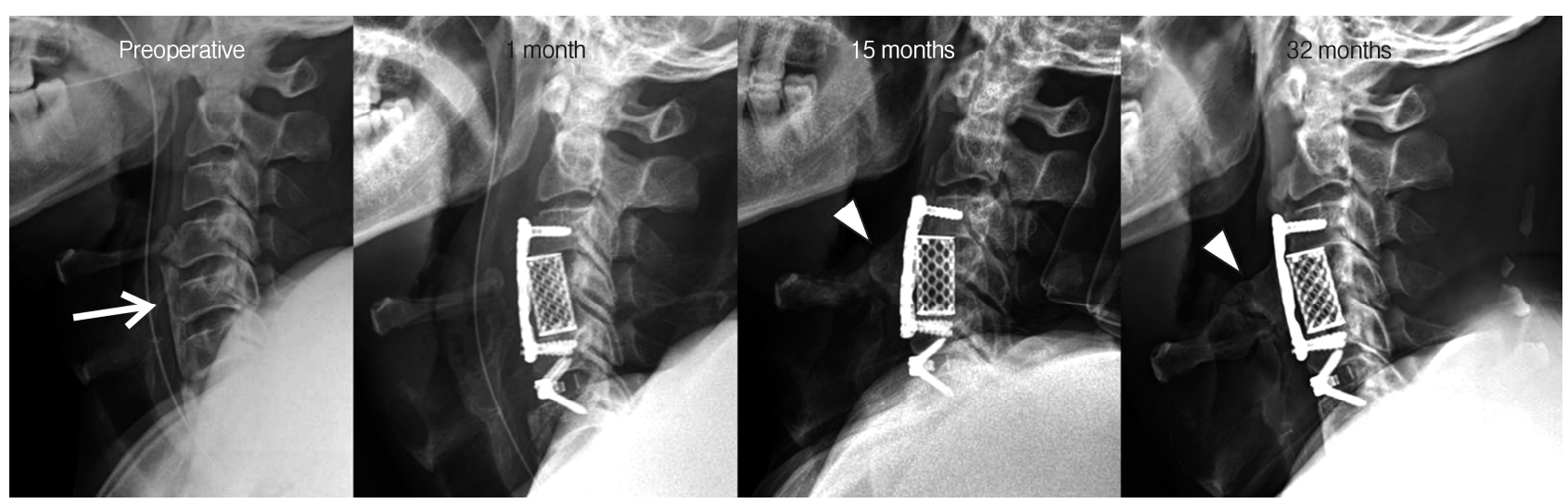

Fig. 2. Chronological changes in the development of heterotopic ossification following anterior cervical fusion in the current patient. Anterior cervical osteophyte (arrow) in the cenvical spine was evident in the pre-operative lateral cervical spine x-ray. Removal of the anterior cervical osteophyte from diffuse idiopathic skeletal hyperostosis is shown in the radiograph taken 1 month post-operatively. However, regrowth of osteophyte is evident radiologically at 15 and 32 months post-operatively.

reported about $30 \%$ to $40 \%$ pain relief at 6 months post-implantation despite maximal medical treatment. His swallowing difficulty did not show any change and annual follow-up was planned.

\section{DISCUSSION}

\section{DISH and Anterior Cenvical Osteophytes}

DISH is a poorly understood, systemic condition characterized by progressive calcification and ossification of ligaments and enthuses ${ }^{8)}$. The formal diagnosis of DISH is established radiologically based on simple descriptive morphological abnormalities of the thoracic spine ${ }^{12,13}$. Although DISH is a largely asymptomatic condition, increasing evidences suggests that DISH was an indicator of several pathological conditions ${ }^{8)}$. The presence of DISH may indicate underlying metabolic derangement and cardiovascular disease. Moreover, bone deposition may lead to biomechanical changes of the musculoskeletal system and the formation of obstructive cervical masses ${ }^{8,17)}$. Several risk factors, including male sex, hypertrophic osteoarthritis, and ankylosing spondylitis have been suggested in the literatures.

The mechanism of swallowing difficulty due to large cervical osteophytes is still unknown. However, a variety of mechanisms have been suggested, including direct mechanical compression of the pharynx and esophagus ${ }^{12)}$, disturbances of normal epiglottis tilt over the laryngeal inlet by the osteophytes at C3 and $\mathrm{C} 4$ level $^{6,14)}$, tissue inflammation around the esophagus ${ }^{1)}$, and cricopharyngeal $\mathrm{spasm}^{1}$. The incidence of dysphagia in patients with DISH ranged between $17 \%$ and $28 \% 0^{9,12)}$. Conservative treatment is indicated for the initial management. Surgical decompression via osteophytectomy is an effective treatment for patients with severe dysphagia and airway obstruc$\operatorname{tion}^{1,3,6,9,11,16,18)}$.
Although simple surgical resection of the osteophytes has been effective in relieving dysphagia, long-term recurrence of osteophytes with dysphagia after 10 years following osteophytectomy has also been reported ${ }^{11)}$. After a mean follow-up period of 9 years (range, 6-13 years) in seven patients with severe dysphagia, who underwent surgical resection of the cervical anterior osteophytes accompanying DISH, radiological recurrence of osteophytes occurred, with an average increase rate of approximately $1 \mathrm{~mm}$ /year following resection ${ }^{11)}$. Two out of seven patients developed recurrent dysphagia along with osteophyte formation ${ }^{11)}$. The incidence of recurrent osteophytes was significantly higher in the 16 segments with preserved mobility than in four segments without mobility ${ }^{11)}$. Because the incidence of recurrent osteophyte is lower in fused intervertebral segments ${ }^{11)}$, studies suggested that osteophytectomy and fusion was effective in relieving dysphagia and recurrence after resection in patients with DISH. However, it is still inconclusive because of small sample size (6 patients) and short follow-up was short ( $23 \pm 8$ months).

\section{HO}

$\mathrm{HO}$ refers to the bone formation outside the skeletal system. In the cervical spine, following fusion or artificial disc replacement, it may consist of ectopic bone formation anywhere around the implant ${ }^{15)}$. The incidence of $\mathrm{HO}$ following cervical TDR was reported as high as $66 \%$ of patients to none ${ }^{15)}$. Tu et al. ${ }^{15)}$ reported about 50\% incidence of $\mathrm{HO}$ with more than $96 \%$ of these levels remaining mobile with no adverse effect on the clinical outcome. Multi-level surgery, male sex, old age, trauma to the longus colli muscle, hypertrophic osteoarthritis, ankylosing spondylitis, extensive bone removal, and DISH have been implicated in the development of $\mathrm{HO}^{15)}$.

Reflecting systemic bone-formation, patients with DISH have a greater propensity to develop $\mathrm{HO}$ in response to local events, 
including joint replacement surgery ${ }^{2,8)}$. Occurrence of HO in joint replacement surgery impairs the function of the affected joint and prophylactic treatment with irradiation or non-steroidal anti-inflammatory drugs may be indicated for such patients $^{2,7,8)}$. It is currently unknown what causes the formation of HO. However, it was suggested that surgical trauma may induce ossification via unknown mechanisms ${ }^{7}$. To prevent $\mathrm{HO}$, ample irrigation and implantation of anterior cervical plate at least $5 \mathrm{~mm}$ away from the adjacent disc space have been suggested for patients with DISH who underwent $\mathrm{ACF}^{5)}$.

\section{CONCLUSION}

An exuberant $\mathrm{HO}$ causing dysphagia 5 years after multilevel $\mathrm{ACF}$ in a patient with DISH is reported. Severe HO and recurrence of cervical osteophyte following osteophytectomy and fusion occurs in patients with DISH and careful long-term followup is warranted.

\section{CONFLICTS OF INTEREST}

No potential conflict of interest relevant to this article was reported.

\section{REFERENCES}

1. Akhtar S, O'Flynn PE, Kelly A, Valentine PM: The management of dysphasia in skeletal hyperostosis. J Laryngol Otol 114:154157, 2000

2. Belanger TA, Rowe DE: Diffuse idiopathic skeletal hyperostosis: musculoskeletal manifestations. J Am Acad Orthop Surg 9:258267, 2001

3. Carlson ML, Archibald DJ, Graner DE, Kasperbauer JL: Surgical management of dysphagia and airway obstruction in patients with prominent ventral cervical osteophytes. Dysphagia 26:34-40, 2011

4. Forestier J, Rotes-Querol J: Senile ankylosing hyperostosis of the spine. Ann Rheum Dis 9:321-330, 1950

5. Kasliwal MK, Tan LA, Wewel JT, Traynelis VC: Exuberant heterotopic ossification following anterior cervical fusion. Neurol India 64:571-572, 2016
6. Lambert JR, Tepperman PS, Jimenez J, Newman A: Cervical spine disease and dysphagia. Four new cases and a review of the literature. Am J Gastroenterol 76:35-40, 1981

7. Langen B, Rudqvist N, Helou K, Forssell-Aronsson E: Microarray studies on 211at administration in $\mathrm{BALB} / \mathrm{C}$ nude mice indicate systemic effects on transcriptional regulation in nonthyroid tissues. J Nucl Med 58:346-353, 2017

8. Mader R, Verlaan JJ, Buskila D: Diffuse idiopathic skeletal hyperostosis: clinical features and pathogenic mechanisms. Nat Rev Rheumatol 9:741-750, 2013

9. Maiuri F, Stella L, Sardo L, Buonamassa S: Dysphagia and dyspnea due to an anterior cervical osteophyte. Arch Orthop Trauma Surg 122:245-247, 2002

10. Mazières B: Diffuse idiopathic skeletal hyperostosis (ForestierRotes-Querol disease): what's new? Joint Bone Spine 80:466470, 2013

11. Miyamoto K, Sugiyama S, Hosoe H, linuma N, Suzuki Y, Shimizu $\mathrm{K}$ : Postsurgical recurrence of osteophytes causing dysphagia in patients with diffuse idiopathic skeletalhyperostosis. Eur Spine J 18:1652-1658, 2009

12. Resnick D, Niwayama G: Radiographic and pathologic features of spinal involvement in diffuse idiopathic skeletal hyperostosis (DISH). Radiology 119:559-568, 1976

13. Resnick D, Shapiro RF, Wiesner KB, Niwayama G, Utsinger PD, Shaul SR: Diffuse idiopathic skeletal hyperostosis (DISH) [ankylosing hyperostosis of Forestier and Rotes-Querol]. Semin Arthritis Rheum 7:153-187, 1978

14. Suzuki K, Ishida Y, Ohmori K: Long term follow-up of diffuse idiopathic skeletal hyperostosis in the cervical spine. Analysis of progression of ossification. Neuroradiology 33:427-431, 1991

15. Tu TH, Wu JC, Huang WC, Guo WY, Wu CL, Shih YH, et al.: Heterotopic ossification after cervical total disc replacement: determination by CT and effects on clinical outcomes. J Neurosurg Spine 14:457-465, 2011

16. Urrutia J, Bono CM: Long-term results of surgical treatment of dysphagia secondary to cervical diffuse idiopathic skeletal hyperostosis. Spine J 9:e13-e17, 2009

17. Verlaan JJ, Boswijk PF, de Ru JA, Dhert WJ, Oner FC: Diffuse idiopathic skeletal hyperostosis of the cervical spine: an underestimated cause of dysphagia and airway obstruction. Spine J 11:1058-1067, 2011

18. von der Hoeh NH, Voelker A, Jarvers JS, Gulow J, Heyde CE: Results after the surgical treatment of anterior cervical hyperostosis causing dysphagia. Eur Spine J 24 Suppl 4:S489-S493, 2015 